\title{
Fog Computing for Trust in the Internet of Things (IoT): A Systematic Literature Review
}

\author{
Kazi Masum Sadique \\ Department of Computer and Systems \\ Sciences \\ Stockholm University \\ Stockholm, Sweden \\ sadique@dsv.su.se
}

\author{
Rahim Rahmani \\ Department of Computer and Systems \\ Sciences \\ Stockholm University \\ Stockholm, Sweden \\ rahim@dsv.su.se
}

\author{
Paul Johannesson \\ Department of Computer and Systems \\ Sciences \\ Stockholm University \\ Stockholm, Sweden \\ pajo@dsv.su.se
}

\begin{abstract}
Fog computing-based solution improves the quality of IoT solutions by making a bridge between the cloud layer and end devices of the IoT paradigm. Fog computing can also increase security and trust in IoT by processing data at the fog layer which is closer to the source of data where it is produced. Reproducibility of research results is one of the curtail criteria for any scientific research. Proper documentation about research methodology allows fellow researchers to reproduce the results and to further extend the research findings. Recent researches are addressing fog computing-based trust and security solutions for the resource constraint IoT devices as it is more convenient and efficient to manage those from the nearest location and not managing those from the cloud. In this paper, we have presented the current state of fog computing-based trust solutions for IoT with future research direction within the area. A framework for trust management for IoT at the fog layer is also proposed in this paper. We have also discussed different scientific research approaches used in fog computing-based trust management in IoT research. The strengths and weaknesses of existing research methodologies used in fog computing-based trust management solutions in the internet of things (IoT) research are also discussed.
\end{abstract}

Keywords- Internet of Things (IoT), Fog Computing, Identity Management, Authentication, Authorization, Trust Management, Security, Privacy.

\section{INTRODUCTION}

Internet of Things (IoT) end devices are mostly used for a specific purpose and are resource constraint devices. It means that end nodes have less computational power. The fog layer is the layer between the cloud layer and the end devices layer is an IoT paradigm [1]. Fog Computing can support the IoT with computation capabilities, enhance network service along with reduced latency, and storage near to end devices of IoT. Fog Computing also works as a middleware between the Cloud and the Edge network. Recent IoT applications and smart services include but not limited to Smart Home, Smart Grid, Smart Farming, Smart Industries, Smart City, Smart Agriculture, Autonomous Vehicles, etc. A typical layered architecture of the Internet of things (IoT) is presented in consists of end devices with less computability, fog devices closer to the end devices and the cloud devices over the fog layer (Fig. 1.). Fog devices play a vital role to keep the IoT end nodes connected with the cloud layer. All these smart IoT services are built upon heterogeneous wireless sensor networks and required more flexibility with location awareness, mobility service, decentralized geographical distribution, real-time communications and immediate decisions which can be achieved by Fog Computing [1][2]. As the huge number of IoT devices will change their locations and communicate with each other in real-time, they need trusted relation between them. For a centralized cloud-based infrastructure it is hard to manage these heterogeneous IoT networks and to keep track of trusted and malicious devices at the edge network. Fog Computing can enhance trust management, security, and privacy of user data near to the origin [1]. To increase trust and security between end nodes, fog computing-based security solutions are useful [1]. Recently many academic researchers are addressing fog computingbased trust management for IoT devices in their research work. As the fog layer has better computability and it is closer to the end devices it is easy to implement security rules and algorithms. Proper documentation of research methodology is important for reproduction and extensions of research findings. Motivated by the above-mentioned reasons, we have performed our research on fog computing for trust in IoT. We have analyzed papers related to trust solutions at the fog layer as well as contributions to research methodologies in those papers.

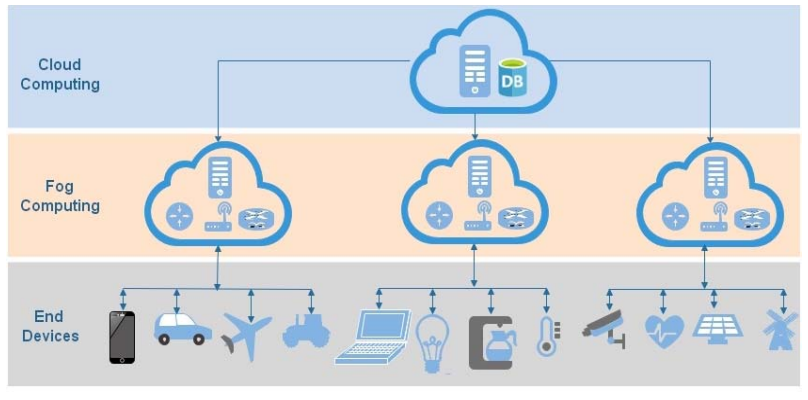

Fig. 1. A typical layered architecture of the Internet of things (IoT)

\section{RESEARCH GOAL AND RESEARCH QUESTIONS}

\section{A. Research Goal}

The goal of this study is to identify different researches in fog computing based trust management solution in internet of things (IoT).

\section{B. Research Question}

The following research questions are answered in this study:

1) How can we improve trust in IoT using Fog Computing?

2) What is the current state of the research in trust solutions at the Fog Layer?

3) What are the methodologies used in fog computing based trust management solution in internet of things (IoT) research?

4) What are the strengths and weaknesses of existing research methodologies used in fog computing based trust management solution in internet of things (IoT) research?

We have answered four research questions in the following sections of this paper: 
- The research question one is answered at the subsections ' $\mathrm{C}$. Research Challenges and Future Directions' and 'D. Proposed Framework' under the 'V. Results' section.

- The research question two is answered at the subsection 'A. Findings from Content Review' under the 'V. Results' section.

- The research question three answered at the subsection 'B. Research Methodology' under the 'V. Results' section.

- The research question four is answered at the subsection 'E. Advances and Disadvantages of the used methods' under the 'V. Results' section.

\section{RELATED WORK}

Several recent researches are addressing trust and security in IoT. In [3], a statistical theory-based trust model for entities on the Internet is presented by the authors. The data analysis is performed based on an example data set. The trust on entity increases or decreases based on the recommendations from other entities. The research has a case study-based design. The paper presented how entities on the internet can measure the trust of another entity it communicates. The trust of any entity is measured based on its' previous experiences and recommendations from other entities. The strengths of the research are that the authors have presented their assumptions and presented real-life examples related to their presented concept. The weakness of this research is the authors have performed a statistical analysis based on assumed data values and, it could defer. The authors in [3], have measured outcome distribution based on any task performed by an entity. They have also calculated standard deviations and estimated the error of a trust value. As an entity becomes trusted based on recommendations and experiences, it could be difficult for a new entity joining to the network to be trusted by others, this could be a problem, but the authors have suggested a solution to it by mapping between spaces also presented histograms to initialized situational trust outcomes. The authors in [3], have performed external validation of their findings based on previous research findings in the decisionmaking section of their paper. The strengths of this research paper are that the authors have presented issues related to the recommendations for any trusted entity, though it is a weakness of their findings. The paper didn't present any evidence of the reliability of their findings. The novelty of this research is, this is one of early research in the field of a trust model for the internet of things/entities where statistical analysis was performed.

A quantitative trust model for supply chains of foods and food safety is presented in [4]. Data-driven trust model is presented in this paper. The Bayesian network and the probability table are used for the trust evaluation in this research [4]. The trust model in this paper is built based on the historical data and facts collected from real-world scenarios, which are the strengths of this research. The authors have considered a quantitative approach for testing their hypotheses and the authors have used meta-analytic research design in [4]. In [5], a model for distributed trust and reputation for peer to peer network is presented. The authors have considered the experimental research strategy in this research. Here internal validity is done because scenarios simulations for the proposed model are performed in this research. In this paper, stability and reliability are measured using a simulation of the designed system. Quantitative data collection is performed by simulation and in performance results and analysis sections quantitative data analysis is performed.

In [6], link-layer security features implementation for low power IoT nodes/devices is presented. The researcher mentioned that the proposed solution is IEEE 802.15.4compliant, and the performance evaluation of the implemented system is also performed. The research strategy is experimental research. Convergent validity is presented in this research. Time and memory requirements analysis are performed in this research. The novelty of this research could be the link-layer security solution for low power devices. In [7], a review research design is followed. The authors have performed a systematic quantitative literature review on privacy principles used in the Internet of Things (IoT) researches. The database selection methods, time range for research article selection, keywords, inclusion and exclusion criteria are discussed in the paper [7]. Any future researcher can further validate the research findings based on the methodology adopted in this research, which is the strength of this research. The authors have specified that they have considered the time range between the years 2009 and 2016, it could be a weakness of this research, as the term Internet of Things (IoT) was introduced by 'Kevin Ashton' during the year 1999 [7]. The validity requirement is discussed in the abstract section of this paper [7]. A qualitative literature review is performed here which represents the research differently as most of the systematic literature reviews are qualitative.

\section{METHODS}

The Systematic literature review is one of the powerful ways for concept development and for finding the research gap in a specific research area [8]. A systematic literature review is performed to identify the data sources for this research study. Details of selection criteria, database selection, search terms, search results, and data analysis methods are described below.

\section{A. Selection criteria}

The following inclusion and exclusion criteria are used for the selection of papers.

\section{1) Inclusion criteria}

- Only journal and conference papers are considered

- Papers are written in English

- Papers which somehow related to fog computingbased trust management solution for IoT

- $\quad$ Papers published as digital format

\section{2) Exclusion criteria}

- Books and other sources

- Papers not written in English 
- Papers not related to fog computing, internet of things and trust management

\section{B. Selected Database}

In this study, four electronic databases are used to search the related articles and proceedings papers. The name of the databases with search fields and the last access date are presented in Table I. Those databases are considered because it was suggested by expert researchers in the area of IoT and Fog Computing.

TABLE I. SElEcted DATABASES

\begin{tabular}{|c|c|c|}
\hline Database & Searched In & $\begin{array}{c}\text { Last } \\
\text { accessed }\end{array}$ \\
\hline $\begin{array}{c}\text { ACM Digital } \\
\text { Library }\end{array}$ & $\begin{array}{c}\text { The ACM Full-Text } \\
\text { Collection }\end{array}$ & 28th April 2019 \\
\hline IEEE Xplore & Metadata only & 28th April 2019 \\
\hline Scopus & $\begin{array}{c}\text { Article title, abstract, } \\
\text { keywords }\end{array}$ & 28th April 2019 \\
\hline $\begin{array}{c}\text { Web of } \\
\text { Science }\end{array}$ & Topic & 28th April 2019 \\
\hline
\end{tabular}

\section{Search Terms}

To search relevant articles advance search option was used for each of the selected databases. The databases were accessed from the Stockholm University Library page. Table II presents the search keywords with the name of the database and search string produced at the database access web page.

TABLE II. DATABASE WITH SEARCH KEYWORD AND THE SEARCH STRING

\begin{tabular}{|c|c|c|}
\hline Database & Search keywords & Search string \\
\hline $\begin{array}{l}\text { ACM } \\
\text { Digital } \\
\text { Library }\end{array}$ & $\begin{array}{l}\text { Internet of Things (IoT), Fog } \\
\text { Computing, Trust }\end{array}$ & $\begin{array}{l}\text { (Internet of things (IoT) } \\
+ \text { +Fog + computing } \\
+ \text { +Trust) }\end{array}$ \\
\hline $\begin{array}{l}\text { IEEE } \\
\text { Xplore }\end{array}$ & $\begin{array}{c}\text { Internet of Things (IoT), Fog } \\
\text { Computing, Trust }\end{array}$ & $\begin{array}{l}\text { (((Internet of things } \\
\text { (IoT)) AND Fog } \\
\text { computing) AND Trust) }\end{array}$ \\
\hline Scopus & $\begin{array}{c}\text { Internet of Things (IoT), Fog } \\
\text { Computing, Trust }\end{array}$ & $\begin{array}{c}\text { (TITLE-ABS- } \\
\text { KEY (internet AND of } \\
\\
\text { AND things ) OR TIT } \\
\text { LE-ABS- } \\
\text { KEY ( iot ) AND TIT } \\
\text { LE-ABS- } \\
\text { KEY ( fog AND comp } \\
\text { uting ) AND TITLE- } \\
\text { ABS- } \\
\text { KEY ( trust ) ) AND D } \\
\text { OCTYPE ( ar OR cp ) } \\
\text { AND ( LIMIT- } \\
\text { TO ( LANGUAGE, "E } \\
\text { nglish" ) ) }\end{array}$ \\
\hline $\begin{array}{l}\text { Web of } \\
\text { Science }\end{array}$ & $\begin{array}{c}\text { Internet of Things (IoT), Fog } \\
\text { Computing, Trust }\end{array}$ & $\begin{array}{l}\text { (TS=(Internet of things } \\
\text { AND Fog computing } \\
\text { and Trust)) AND } \\
\text { LANGUAGE: (English) } \\
\text { AND DOCUMENT } \\
\text { TYPES: (Article) }\end{array}$ \\
\hline
\end{tabular}

\section{Search Result}

From the search results, we have considered all the papers at the first iteration. We have removed the redundant results in our next iteration. The number of papers found in different databases in the first state is presented in Table III.
TABLE III. SELECTED NUMBER OF PAPERS

\begin{tabular}{|c|c|}
\hline Database & $\begin{array}{c}\text { Number of identified pa-pers } \\
\text { (including duplicates) }\end{array}$ \\
\hline ACM Digital Library & 7 \\
\hline IEEE Xplore & 27 \\
\hline Scopus & 26 \\
\hline Web of Science & 9 \\
\hline
\end{tabular}

\section{E. Selection based on Keyword Frequency and Content Review}

A Total of fifty-six papers (excluding duplicates) were identified after the first iteration of selection in four different databases. In the second iteration, the documents were checked for the frequency of the keyword used. At this stage, the number of paper reduces to 31 . In the last round of selection, documents were thoroughly checked, and 15 papers were selected. The number of finally selected papers with their research methodology is presented in Table IV.

TABLE IV. IDENTIFIED PAPERS BASED ON RESEARCH METHODOLOGY

\begin{tabular}{|c|c|}
\hline Research Method & Number of identified papers \\
\hline Qualitative method & 3 \\
\hline Quantitative method & 10 \\
\hline Mixed method & 2 \\
\hline
\end{tabular}

\section{F. Data Collection and Analysis}

The primary data sources of this study are the 15 selected papers. Selected papers are reviewed on different criteria: research strategy, data collection method, and data analysis method. The research strategies are considered from [9]: surveys, case studies, experiments, ethnography, phenomenology, grounded theory, action research, and mixed methods. Selected papers are also reviewed for data collection methods and data analysis methods considering the methods described by Denscombe [9].

\section{G. Limitations}

In this study four popular databases are selected for literature search, it might exclude some of the relevant literature. Another limitation is only conference and journal papers written in English are included in this study.

\section{RESULTS}

In this study, a total of 15 papers related to fog computing-based trust management in IoT are reviewed.

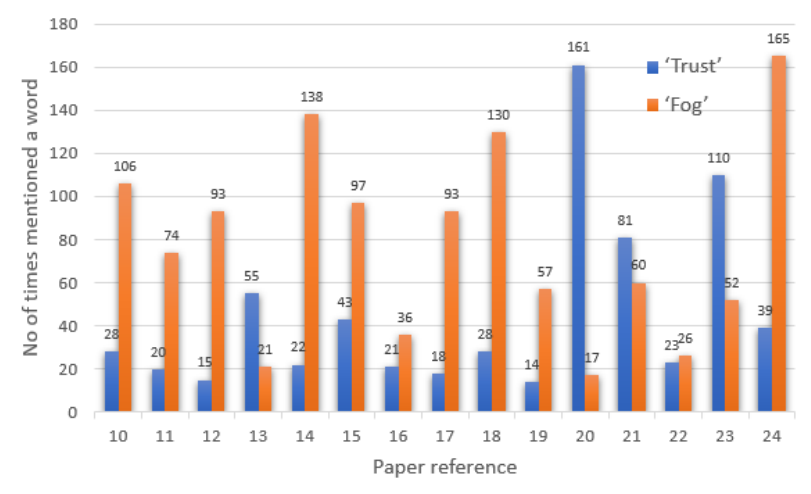

Fig. 2. Frequency of use of trust and fog in selected papers 
The frequency of use of the words 'Trust' and 'Fog' in the text in the selected papers are shown in the Fig. 2. The highest no of use of 'Trust' is 161 and the lowest number of uses of 'Trust is 14. The highest number of times use of the word 'Fog' is 165, the lowest number of uses of 'Fog' is 17 within the 15 selected papers.

\section{A. Findings from Content Review}

From our detailed content review, we have identified several key points while considering trust in IoT via the fog layer. Identified key points are described below:

- Secure Communication: For the enhancement of the trusted relationship between the IoT devices as well as the IoT gateway, secure communication between those are essential [10][12]. Fog based secure communication schemes proposed in [10], [12].

- Key Management: A trusted way of key management is also essential. A secure key management scheme can increase security and trust in the IoT paradigm [10],[11].

- Secure Data Storage: Secure data storage at the fog layer for the huge IoT devices can also improve the trust and security in IoT. Distributed secure data storage with data synchronization capabilities is proposed in [11]. Multi-level trusted implementation with the use of collaborative computing can enhance the security of data storage at the fog layer [11].

- Lightweight Security Algorithms: The design and implementation of lightweight security algorithms at the fog layer are essential for resource constraint IoT end devices [12], [13]. A fog-based architecture with proxy re-encryption and publish/subscribe communication model for secure communication with low power devices is proposed in [12].

- Authentication of devices: Secure authentication of IoT devices, as well as user devices, is another key point [13]. A fog layer authentication mechanism can enhance trust and security in the IoT with quick support to the IoT devices without interaction with the cloud layer.

- Authorization and/or Access Control: Only authorized devices will have access to a certain resource within the network. Authorization algorithms implemented at fog layer also enhance trust in IoT [13], [16], [17].

- Data Privacy: As the IoT devices collect data from society a privacy-preserving technique is also crucial. A fog layer implementation of a privacy mechanism can enhance the confidentially of user data [14]-[16], [23], [24].

- Identity Management: Identity management of huge IoT devices can be handled at the fog layer to enhance the trust level between IoT end devices as well as communication with applications implemented at the cloud layer [18].

- Intrusion Prevention System (IPS): Implementation of the intrusion prevention system [19] at the fog layer with machine learning algorithms also enhances trust and security in IoT.

- Trustworthiness: There are different terms related to trust: green/environmental trust, self-trust, social trust, and QoS trust are introduced in [20] which are valid for trust management. A fuzzy logic-based trust evaluation is presented in [21]. A reputationbased trust evaluation model is proposed in [23].

- Decision Making: Fog layer decision making can improve trust in IoT [22]. Fog layer decision support can increase security in IoT as well as improve the quality of service concerning time while communication to the cloud layer is expensive and hard to process data in resource constraint IoT devices.

\section{B. Research Methodology}

Ten of the papers have followed the quantitative research methodology. Three papers have used the qualitative research method and two papers have used both qualitative and quantitative research methods, namely mixed methods. Table V presents the result of identified papers based on research strategy, data collection method and data analysis method.

TABLE V. LITERATURE REVIEW BASED ON RESEARCH METHODOLOGY

\begin{tabular}{|c|l|l|l|}
\hline $\begin{array}{l}\text { Paper } \\
\text { Reference }\end{array}$ & $\begin{array}{l}\text { Research } \\
\text { Strategy }\end{array}$ & $\begin{array}{l}\text { Source/Method } \\
\text { of Data } \\
\text { Collection }\end{array}$ & $\begin{array}{l}\text { Method of Data } \\
\text { Analysis }\end{array}$ \\
\hline $\begin{array}{c}{[10],[11],} \\
{[20],[21],} \\
{[22]}\end{array}$ & Experiments & Simulations & Quantitative \\
\hline $\begin{array}{c}{[12],[15],} \\
{[17],[19]}\end{array}$ & Experiments & Testbed & Quantitative \\
\hline $\begin{array}{c}{[13],[16],} \\
{[18]}\end{array}$ & $\begin{array}{c}\text { Literature } \\
\text { review }\end{array}$ & Documents & Qualitative \\
\hline$[14]$ & $\begin{array}{c}\text { Experiments } \\
\text { Real-world smart } \\
\text { metering data set }\end{array}$ & Quantitative \\
\hline$[23]$ & Experiments & $\begin{array}{c}\text { Observation/ } \\
\text { Simulations }\end{array}$ & Mixed method \\
\hline$[24]$ & Experiments & $\begin{array}{c}\text { Observation/ } \\
\text { Testbed }\end{array}$ & Mixed method \\
\hline
\end{tabular}

One of the strong research strategies for concept development is the literature review [25]. In [13], [16], [18] literature review research strategy is used. These three papers [13], [16], [18] have considered qualitative data analysis. Only two of the selected papers [23, [24] have used mixed data analysis method. Though there was no limit assigned for the publication year most of the identified papers are published between the years 2017 and 2019. Research findings need to be reproducible. A clear method section may increase the quality of a paper with respect to the validity and reproducibility of a similar result [8], [9]. IoT is improving the quality of human life every day with new innovative contribution and it is becoming more part of humanities and social science, so a clear presentation of the ethical and method section is becoming crucial. None of the reviewed papers have discussed ethics and demonstrated ethical consideration for trust management for socio- 
technology-based systems. Privacy is one of ethical issue which has been discussed in papers [14]-[16], [23], [24].

\section{Research Challenges and Future Directions}

As we have mentioned in our background and motivation section, IoT sensors and actuators are mostly resource constraint devices. Fog layer can support these end devices with better computation capabilities and storage. But we still need to take care of the trust and security issue in between these devices (end device to end device) as well as their communication with the Fog devices. To ensure trust and security for these small devices, context aware solution can be used with lightweight encryption schemes can be implemented at the resource constraint devices which will further communicate securely with the Fog Layer of IoT [10], [12], [13]. Most of the recent researches are considering authentication and authorization as a challenge for huge number of IoT devices with enormous amount of data generated from the society [13], [16], [17]. There is a big challenge in how devices at the fog layer and IoT end devices can authenticate themselves efficiently, securely and anonymously. The trust management of heterogeneous IoT devices is an also a big challenge which can possibly solve by using decentralized control at the fog layer. Identity management [18] of huge number of IoT devices is another challenge which needs to be addressed locally at the Fog Layer. The fog layer network components must share data and cardinals of the devices located locally but it must be as per requirement. Fog layer devices needs to be trusted by the end devices as well as cloud devices. Trusted computing or trusted execution environment (TEE) concept [26] can be implemented at the fog layer devices to increase trust between different components of IoT from the edge to the cloud.

\section{Proposed Framework}

Based on the identified challenges we have designed a framework for trust management module which can be implemented at the fog layer of IoT paradigm.

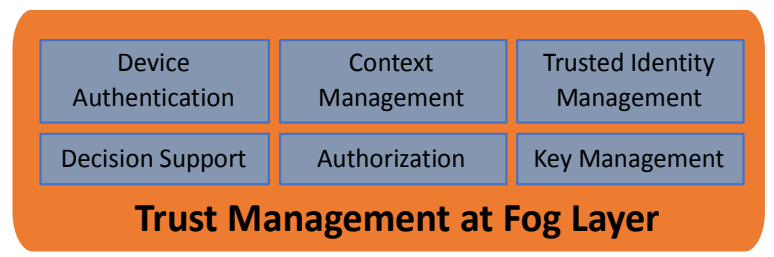

Fig. 3. Fog layer Trust Management of the Internet of things (IoT)

There are six components in the trust management module for IoT. All six components (Fig. 3.) together support the IoT end devices with trust and security services. We have described the functions of each component below:

- Device Authentication: The IoT end device authentication should be performed in this module. Device authentication block will work together with the trusted identity management block for authentication of IoT devices locally without sending an authentication request to the cloud layer. As the devices are authenticated locally and verified by the trusted identity management block, a trust rating should be added against the identity of the entity. This will reduce network traffic as well as increase the efficiency of the system. We should be able to avoid possible eavesdropping on the identity-related cardinals of an entity that can happen during an authentication from a global cloud device.

- Context Management: The context management block keeps track of context information of end devices connected at the edge layer. For example, information related to IoT device movement, location information, time, reputation and so on. This block will be able to enhance the trust management as it will keep context information locally at the fog layer. It will also reduce unnecessary traffic towards the cloud as the context data will be stored locally.

- Trusted Identity Management: Trusted Identity Management block will keep track of the logical identity of end devices locally. This block will support the authentication and authorization module with the requested information. This module will continuously improve its database with supporting information collected from the decision support block, context management block and key management block.

- Decision Support: The decision support block supports all the five other blocks with quick decision making based on the requirement. Decisions could be related to the rating of an entity, authentication, and authorization of device or key management or update of logical keys at the key management block. It will also support with a quick decision to resource constraint devices which are running time-critical applications.

- Authorization: The authorization block allows or denies access to services or resources based on the authorization rights assigned to that specific IoT device. This block will also increase trust between the devices because an authorization table could be updated from the rating stored in the trusted identity management block based on the early activities of a device within the network.

- Key Management: The key management block also a part of the trust management at the fog layer. This block will track the keys of connected devices at the edge layer. For secure interaction between devices, cryptography is essential. The resource constraint devices will not be able to store thousands of keys of devices. The key management block will store the keys of trusted end devices only.

\section{E. Advantages and Disadvantages of used Methods}

In paper [21], authors have discussed the fuzzy logic method to evaluate trust in fog computing. Fuzzy logic and fuzzy sets are terms used in artificial intelligence. Fuzzy logic is applied to fuzzy sets for reasoning with logical expressions. Fuzzy sets present vagueness of objects [21]. Three of the identified papers have literature review research strategies, but the authors of the papers didn't consider a systematic literature review, which could be a weakness of their research, in case they miss some related publication. Every year many contradictory research findings are published due to lack of systematic literature review [8]. On 
the other hand, a literature survey/review paper without a clear method definition is less/not trustable, in case the authors of the survey paper include some non-peer reviewed low-quality research paper as a data source. Several papers [10]-[12],[14],[15],[17],,[19]-[24] have experiments as research strategy and have used simulations [10],[11],[20][23] and testbed [12],[15],[17],[19],[24] for data collection to test the performance of their proposed models/theory which is good but in some cases, the real-life experiment results differ from the simulation result. It is always good to have a plan to test any designed prototype in a real-life test environment (of course considering safety issues) before implementing it in a live environment. In [14] real-world smart metering data are analyzed. In [23],[24] data analysis method is mixed and here observation is also used as one of the data collection methods. The planned research area is to increase trust between different IoT components in the lower layer and edge layer of the IoT paradigm using fog computing. The literature review research strategy can be useful for concept development within the area of research. On the other hand, data collection by simulation and testbed could be interesting for future research within the abovementioned area of research.

\section{CONCLUSION}

In this paper, we have addressed the current research status of trust solutions for the Internet of Things at the Fog layer also proposed a model for trust management and suggested some future research directions. Several key points for trust enhancement at the fog layer of the IoT paradigm are discussed in this paper. It is observed that there is a need for standards at the architectural level for trust management at the fog layer of IoT for better governance. There are various technologies which have been used for the trust and security analysis purpose. But many challenges need to address and will be resolved in the future by purposing new models and algorithms for trust and security management at the fog layer in IoT paradigm.

\section{REFERENCES}

[1] I. Stojmenovic and S. Wen, "The Fog computing paradigm: Scenarios and security issues," 2014 Fed. Conf. Comput. Sci. Inf. Syst. FedCSIS 2014, vol. 2, pp. 1-8, 2014, doi: 10.15439/2014F503.

[2] F. Bonomi, R. Milito, J. Zhu, and S. Addepalli, "Fog computing and its role in the internet of things," Proc. first Ed. MCC Work. Mob. cloud Comput. - MCC '12, p. 13, 2012, doi: 10.1145/2342509.2342513.

[3] J. Shi, G. V. Bochmann, and C. Adams, "A trust model with statistical foundation,” IFIP Adv. Inf. Commun. Technol., vol. 173, pp. 145-158, 2005, doi: 10.1007/0-387-24098-5_11.

[4] W. Han, Y. Gu, Y. Zhang, and L. Zheng, "Data driven quantitative trust model for the Internet of Agricultural Things," 2014 Int. Conf. Internet Things, IOT 2014, pp. 31-36, 2014, doi: 10.1109/IOT.2014.7030111.

[5] B. Qureshi, G. Min, and D. Kouvatsos, "A distributed reputation and trust management scheme for mobile peer-to-peer networks," Comput. Commun., vol. 35, no. 5, pp. 608-618, 2012, doi: 10.1016/j.comcom.2011.07.008.

[6] D. Altolini, V. Lakkundi, N. Bui, C. Tapparello, and M. Rossi, "Low power link layer security for IoT: Implementation and performance analysis," 2013 9th Int. Wirel. Commun. Mob. Comput. Conf. IWCMC 2013, pp. 919-925, 2013, doi: 10.1109/IWCMC.2013.6583680.
[7] N. Aleisa and K. Renaud, "Privacy of the Internet of Things: A Systematic Literature Review," pp. 5947-5956, 2016, doi: 10.24251/HICSS.2017.717.

[8] P. Brereton, B. A. Kitchenham, D. Budgen, M. Turner, and M. Khalil, "Lessons from applying the systematic literature review process within the software engineering domain," J. Syst. Softw., vol. 80, no. 4, pp. 571-583, 2007, doi: 10.1016/j.jss.2006.07.009.

[9] M. Denscombe, The good research guide for small-scale social research projects, Buckingham: Open University Press, 1998

[10] A. Ben Amor, M. Abid, and A. Meddeb, "A secure fog-based communication scheme," 2017 Int. Conf. Internet Things, Embed. Syst. Commun. IINTEC 2017 - Proc., vol. 2018-Janua, pp. 146-151, 2018, doi: 10.1109/IINTEC.2017.8325929.

[11] S. He, B. Cheng, H. Wang, X. Xiao, Y. Cao, and J. Chen, "Data security storage model for fog computing in large-scale IoT application," INFOCOM 2018 - IEEE Conf. Comput. Commun. Work., pp. 39-44, 2018, doi: 10.1109/INFCOMW.2018.8406927.

[12] L. Ferretti, M. Marchetti, and M. Colajanni, "Fog-based secure communications for low-power IoT devices," ACM Trans. Internet Technol., vol. 19, no. 2, 2019, doi: 10.1145/3284554.

[13] P. Abhijit J and D. G. Syam Prasad, "Trust Based Security Model for IoT and Fog based Applications.," Int. J. Eng. Technol., vol. 7, no. 2.7, p. 691, 2018, doi: 10.14419/ijet.v7i2.7.10924.

[14] L. Lyu et al., "PPFA : Privacy Preserving Fog-Enabled Aggregation in Smart Grid," IEEE Trans. Ind. Informatics, vol. 14, no. 8, pp. 3733-3744, 2018, doi: 10.1109/TII.2018.2803782.

[15] A. M. Elmisery, S. Rho, and D. Botvich, "A fog based middleware for automated compliance with OECD privacy principles in internet of healthcare things," IEEE Access, vol. 4, no. Idc, pp. 8418-8441, 2016, doi: 10.1109/ACCESS.2016.2631546.

[16] M. Gupta and R. Sandhu, "Authorization framework for secure cloud assisted connected cars and vehicular internet of things," Proc. ACM Symp. Access Control Model. Technol. SACMAT, pp. 193-204, 2018, doi: 10.1145/3205977.3205994.

[17] Q. Xu, C. Tan, Z. Fan, W. Zhu, Y. Xiao, and F. Cheng, "Secure data access control for fog computing based on multi-authority attributebased signcryption with computation outsourcing and attribute revocation," Sensors (Switzerland), vol. 18, no. 5, 2018, doi: $10.3390 / \mathrm{s} 18051609$.

[18] B. A. Martin et al., "OpenFog security requirements and approaches," 2017 IEEE Fog World Congr. FWC 2017, pp. 1-6, 2018, doi: 10.1109/FWC.2017.8368537.

[19] M. Kahla, M. Azab, and A. Mansour, "Secure, Resilient, and SelfConfiguring Fog Architecture for Untrustworthy IoT Environments," Proc. - 17th IEEE Int. Conf. Trust. Secur. Priv. Comput. Commun. 12th IEEE Int. Conf. Big Data Sci. Eng. Trust. 2018, pp. 49-54, 2018, doi: 10.1109/TrustCom/BigDataSE.2018.00018.

[20] N. Kumar, S. Mitra, M. Bhattacharjee, and L. Mandal, "SGSQoT: A Community-Based Trust Management Scheme in Internet of Things," vol. 811. Springer Singapore, 2019.

[21] F. H. Rahman, T. W. Au, S. H. Shah Newaz, and W. S. Suhaili, "Trustworthiness in fog: A fuzzy approach," ACM Int. Conf. Proceeding Ser., pp. 207-211, 2017, doi: $10.1145 / 3171592.3171606$.

[22] D. Arya and M. Dave, "Security-based Service Broker Policy for Fog Computing Environment", $8^{\text {th }}$ ICCCNT, Delhi. 2017.

[23] S. Y. B, TRFIoT: Trust and Reputation Model for Fog-based IoT, vol. 11068. Springer International Publishing, 2018.

[24] T. D. Dang and D. Hoang, "A data protection model for fog computing," 2017 2nd Int. Conf. Fog Mob. Edge Comput. FMEC 2017, pp. 32-38, 2017, doi: 10.1109/FMEC.2017.7946404.

[25] J. M. Cowell, "Literature Reviews as a Research Strategy," The Journal of School Nursing, pp. 326-327, 2012, https://doi.org/10.1177/1059840512458666

[26] B. McGillion, T. Dettenborn, T. Nyman, and N. Asokan, "OpenTEE - An open virtual trusted execution environment," Proc. - 14th IEEE Int. Conf. Trust. Secur. Priv. Comput. Commun. Trust. 2015, vol. 1, pp. 400-407, 2015, doi: 10.1109/Trustcom.2015.400. 